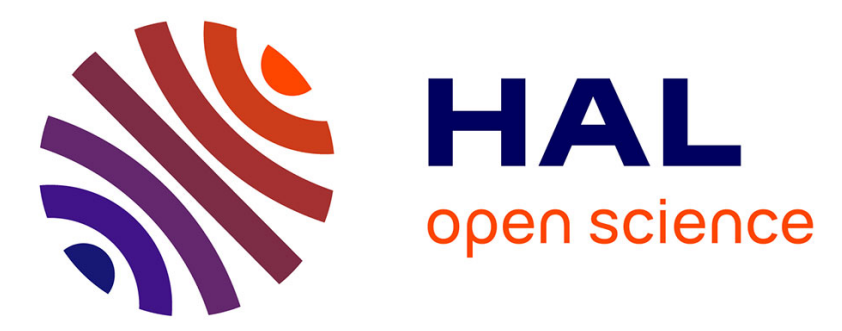

\title{
Désenglacement du haut bassin versant du Vorz Massif de Belledonne, Isère, au Tardiglaciaire et à l'Holocène
}

Ludovic Ravanel, Françoise Allignol, Philip Deline

\section{To cite this version:}

Ludovic Ravanel, Françoise Allignol, Philip Deline. Désenglacement du haut bassin versant du Vorz Massif de Belledonne, Isère, au Tardiglaciaire et à l'Holocène. Neige et glace de montagne: Reconstitution, dynamique, pratiques, Collection EDYTEM - Cahiers de Géographie, nº, pp.23-37, 2009.

halsde-00399777

\section{HAL Id: halsde-00399777 \\ https://hal.science/halsde-00399777}

Submitted on 30 Mar 2011

HAL is a multi-disciplinary open access archive for the deposit and dissemination of scientific research documents, whether they are published or not. The documents may come from teaching and research institutions in France or abroad, or from public or private research centers.
L'archive ouverte pluridisciplinaire HAL, est destinée au dépôt et à la diffusion de documents scientifiques de niveau recherche, publiés ou non, émanant des établissements d'enseignement et de recherche français ou étrangers, des laboratoires publics ou privés. 


\section{Collection EDYTEM}

Numéro 8 - Année 2009

Cahiers de

Géographie

\section{NEIGE et GLACE de MONTAGNE}

Reconstitution, dynamique, pratiques

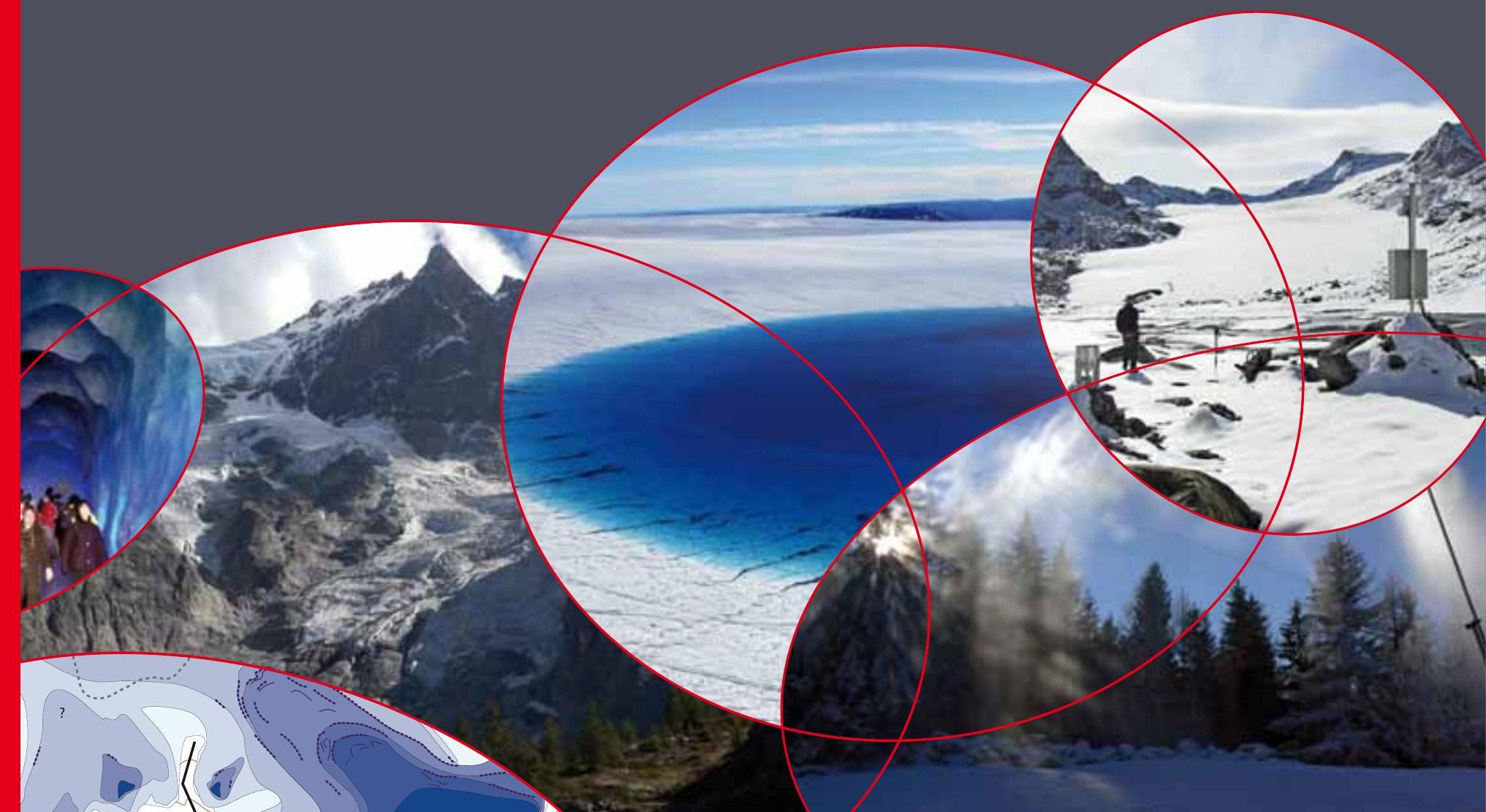





\title{
DÉSENGLACEMENT DU HAUT BASSIN VERSANT DU VORZ MASSIF DE BELLEDONNE, ISÈRE AU TARDIGLACIAIRE ET À L'HOLOCÈNE
}

\author{
LATEGLACIAL AND HOLOCENE GLACIER RETREAT IN THE UPPER VORZ WATERSHED \\ BELLEDONNE MASSIF, FRANCE
}

Ludovic RAVANEL, Françoise ALLIGNOL, Philip DELINE

Laboratoire EDYTEM, Université de Savoie/CNRS, Campus scientifique, F 73376 Le Bourget-du-Lac cedex.

Contact : ludovic.ravanel@univ-savoie.fr

\begin{abstract}
RÉSUMÉ
La reconnaissance et la cartographie des héritages glaciaires dans le haut bassin versant du Vorz (massif de Belledonne, Alpes occidentales françaises) permettent de proposer une chronologie du désenglacement tardiglaciaire et holocène. L'englacement du secteur est aujourd'hui quasiment nul : seuls demeurent le glacier de Freydane-le plus grand du massif $\left(0,3 \mathrm{~km}^{2}\right)$ - et le minuscule glacier relique de Roche Noire. En aval du front actuel de ces deux glaciers, treize positions de front glaciaire ont été identifiées. Des cinq positions holocènes, les quatre dernières datent vraisemblablement de la fin du Petit Âge Glaciaire (vers 1850), de 1890, de la fin des années 1920 et du début des années 1980. En aval des complexes morainiques de l'Holocène-PAG, la cartographie des moraines suggère huit positions tardiglaciaires. Parmi les formes et formations périglaciaires et gravitaires qui recouvrent plus ou moins les moraines, cette étude a identifié quatre écroulements rocheux, d'âge et de dimensions divers. Le principal écroulement (plusieurs millions de $\mathrm{m}^{3}$ ) pourrait dater de la fin du Tardiglaciaire ou du début de l'Holocène et résulter de la disparition d'un paléo-sommet qui était situé sur la crête entre Roche Rousse et Roche Noire.
\end{abstract}

Mots-Clés : DÉSENGLACEMENT, MORAINE, ÉCROULEMENT ROCHEUX, TARDIGLACIAIRE, HOLOCĖNE, CARTOGRAPHIE GÉOMORPHOLOGIQUE, MASSIF DE BELLEDONNE.

\begin{abstract}
Identification and mapping of glacial heritages in the upper Vorz watershed (Belledonne massif, Western French Alps) have provided a chronology of the glacier retreat during the Lateglacial and the Holocene. The glacier retreat in this area is now largely achieved: it just remains the Freydane glacier, the largest in the massif $\left(0.3 \mathrm{~km}^{2}\right)$, and the tiny relict glacier of Roche Noire. Downstream the present fronts of these two glaciers, thirteen positions were recognized. The last four among the five Holocene positions correspond probably to the end of the Little Ice Age (c.1850), 1890, late 1920s and early 1980s. Beyond the morainic complex of the Holocene-LIA, the mapping of moraines suggests eight Lateglacial positions. Among the periglacial and gravitative forms and formations which cover more or less the moraines, there are four very different rockfalls or rock avalanches, of which the largest (at least several millions $\mathrm{m}^{3}$ ) could have occurred at the end of the Lateglacial or at the beginning of the Holocene, and probably corresponds to the collapse of a palaeo-summit from the crest line between Roche Rousse and Roche Noire.
\end{abstract}

KEYWORDS: GLACIER RETREAT, MORAINE, ROCK AVALANCHE, LATEGLACIAL, HOLOCENE, GEOMORPHOLOGICAL MAP, BELLEDONNE MASSIF 


\section{INTRODUCTION}

Depuis le Dernier Maximum Glaciaire, les glaciers ont connu de très nombreuses fluctuations. Les récurrences et les stagnations glaciaires se traduisent dans le paysage par des cordons morainiques plus ou moins bien conservés en fonction de leur âge et des agents d'érosion qui les remanient. Ces cordons peuvent constituer un précieux indicateur de l'évolution climatique en milieu de montagne malgré la complexité de la dynamique des glaciers. De ces fluctuations glaciaires dépend également l'évolution géomorphologique des bassins versants de montagne, jusqu'à leur dynamique actuelle.
Sur la base de l'étude des formes et formations géomorphologiques du haut bassin versant du Vorz (massif de Belledonne), cet article propose d'établir une chronologie du désenglacement d'un haut bassin dans un massif pour lequel on ne dispose que de données parcellaires pour la fin du Tardiglaciaire et l'Holocène : à notre connaissances, seuls Hanss et Wegmüller (1976) et Chardon (1993) ont abordé cette question. La reconstitution morphodynamique et paléogéographique des secteurs étudiés prend également en compte les écroulements, dont les dépôts marquent fortement le paysage du haut Vorz.

\section{I - CADRe GÉOgRAPHIQUe du haUt Vorz}

Le Vorz draine une des vallées du flanc occidental de Belledonne (figure 1). Ce massif cristallin externe est l'un des plus soumis aux influences océaniques. $\mathrm{Au}$ fond du bassin du Vorz, bien que ne couvrant plus qu'une surface très réduite $\left(0,3 \mathrm{~km}^{2}\right)$, le glacier de Freydane constitue actuellement l'appareil glaciaire le plus étendu de Belledonne.

\section{1 - Les secteurs d'étude}

Orienté NE-SW, le massif de Belledonne est délimité par le Grésivaudan à l'ouest, la Combe de Savoie et la vallée de la Maurienne au nord, la vallée du Glandon à l'est et celles de l'Eau d'Olle et de la Romanche au sud. Il s'étire sur $70 \mathrm{~km}$ de long et une quinzaine de kilomètres de large en moyenne. Cette étude porte sur le haut bassin versant du Vorz, avec les secteurs du vallon de la Pierre et du cirque du Lac Blanc, tous deux situés au NNW du Grand Pic (2977 m), point culminant du massif de Belledonne. Ces secteurs appartiennent à l'unité géologique du Rameau Interne du massif de Belledonne. Elle est constituée de matériel anté-alpin métamorphisé au cours de la formation de la chaîne hercynienne (400-300 Ma), composé par trois litholo- gies (Fernandez et al., 2002 ; Hippolyte et al., 2006 ; Guillot et Menot, 2009) : ophiolites, gneiss et leptynites, amphibolites. Les amphibolites (muraille des Trois Pics de Belledonne par exemple) reposent sur des gneiss qui affleurent très largement dans tout le haut bassin (Lac Blanc, Rocher de l'Homme). Ces gneiss, généralement moins résistants que les amphibolites, ont été largement entaillés par l'érosion. Les nombreux héritages glaciaires, dont l'ombilic du Lac Blanc et le complexe morainique du glacier de Freydane sont les meilleurs exemples, attestent du rôle important des glaciers dans ce travail d'érosion. De nombreuses formations morainiques, situées en amont et en aval des moraines du Petit Âge Glaciaire (PAG), témoignent des fluctuations des glaciers dans ces secteurs durant le Tardiglaciaire et l'Holocène.

\section{2 - Contexte glaciaire actuel}

Dans la zone d'étude ne persistent plus aujourd'hui que deux glaciers, cantonnés en pied de paroi et dont l'extension est très différente. Leur persistance n'est due qu'à un contexte local favorable :

- des cônes de neige au contact entre parois et gla-
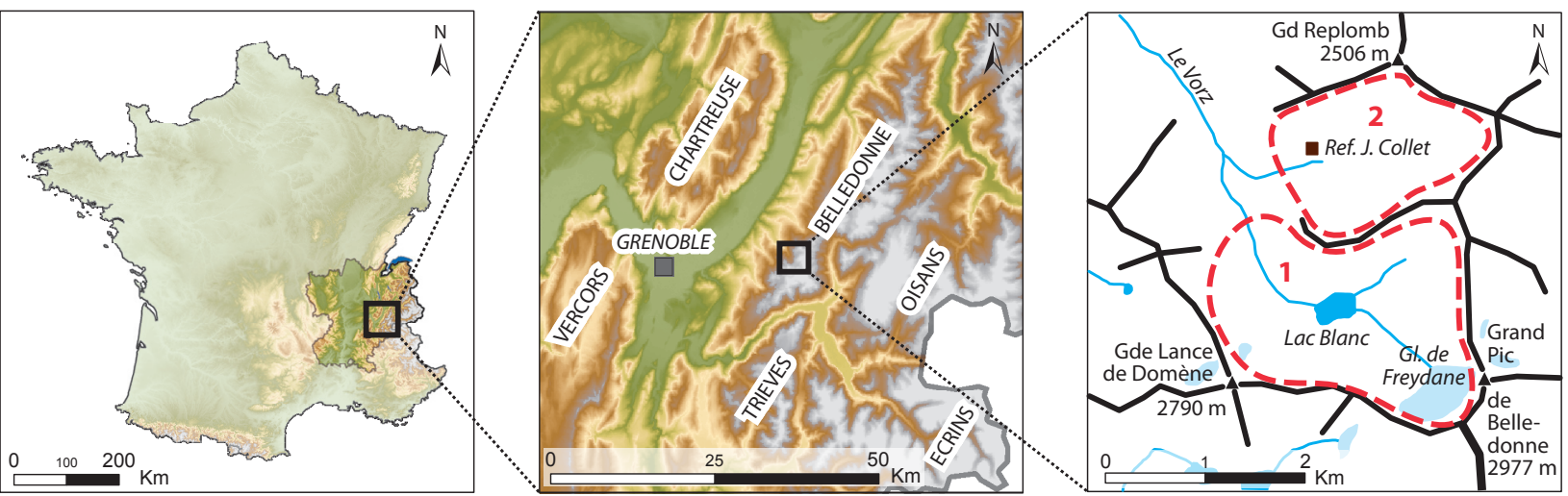

Figure 1 - Localisation du secteur d'étude (1 : cirque du Lac Blanc; 2 : vallon de la Pierre). 
ciers montrent le rôle des avalanches dans l'alimentation de ceux-ci ;

- l'ombre portée des parois assure un microclimat plus froid.

$\mathrm{Au}$ fond du bassin du Vorz, au pied de la muraille de $400 \mathrm{~m}$ de commandement qui culmine au Grand Pic de Belledonne, le glacier de Freydane - dont les altitudes moyenne, minimale et maximale sont respectivement $2530 \mathrm{~m}, 2450 \mathrm{~m}$ et $2620 \mathrm{~m}$ et dont la zone d'accumulation est actuellement quasi inexistante - profite d'une exposition NNW pour maintenir ses $0,3 \mathrm{~km}^{2}$ de surface englacée. En exposition NNW également, à 2610 m d'altitude, le glacier au fond du Ravin de la Lauzière de Roche Noire, en pied de versant, est devenu relique suite certainement à l'été caniculaire 2003. Enfin, quelques névés occupent ponctuellement certains couloirs d'avalanche exposés au nord, à l'ombre des parois. Ces éléments montrent que la déglaciation est quasiment achevée dans l'un des derniers secteurs encore englacés de Belledonne.

\section{II - IDENTIFICATION ET CARTOGRAPHIE DES HÉRITAGES GLACIAIRES}

La carte géomorphologique du vallon de la Pierre et du cirque du Lac Blanc (figure 2) a été réalisée à partir d'observations effectuées lors de campagnes de terrain (juin 2007 et septembre/octobre 2008), dans le cadre d'une étude pluridisciplinaire destinée à améliorer la connaissance du fonctionnement global du torrent du Vorz (Allignol, 2008) après la crue dévastatrice des 22 et 23 août 2005 (Arrêté de reconnaissance de catastrophe naturelle du 06 octobre 2005). Afin de couvrir l'ensemble des secteurs considérés, ces observations ont été complétées par la photo-interprétation d'orthophotos de 2003 et d'aérophotographies de 2007.

Les processus d'érosion glaciaire et les formes d'ablation qui leur sont liées dominent généralement dans le secteur amont des bassins glaciaires, tandis que processus et formes d'accumulation caractérisent l'aval (Campy et Macaire, 2003). Mais dans le haut bassin du Vorz, en raison du retrait quasiment achevé des glaciers, ces deux secteurs sont aujourd'hui confondus. De surcroît, des dépôts d'écroulement, qui peuvent parfois s'apparenter à des formations morainiques (Deline et Kirkbride, 2008), peuvent rendre difficile la reconnaissance des héritages glaciaires.

\section{1 - Un poli glaciaire souvent bien conservé}

A la suite du retrait des glaciers, les paysages du haut bassin du Vorz présentent de nombreuses formes d'érosion glaciaire, particulièrement marquées dans le cirque du Lac Blanc, qui contrastent avec les crêtes acérées et les versants déchiquetés. La forme générale $\mathrm{du}$ fond des vallons est celle d'auges aux bords lisses, surmontées par des cirques glaciaires. Un épaulement, assez peu prononcé, marque la transition entre les auges et les secteurs supérieurs à forte rugosité (secteur de la Grande Lance de Domène, par exemple). Les secteurs étudiés présentent également un profil longitudinal en verrous (verrou du Lac Blanc) et ombilics dans lesquels peuvent se former des lacs comme le Lac Blanc (figure 3). Le niveau du verrou du Lac Blanc est par ailleurs rehaussé par des moraines frontales et des dépôts d'écroulement.
Outre ces macroformes glaciaires, des mésoformes sont facilement identifiables. En particulier, les secteurs du refuge J. Collet, du verrou du Lac Blanc, du Ravin de la Lauzière de Roche Noire et du revers ouest de Roche Noire sont jalonnés de nombreuses roches moutonnées (figure 2). Des microformes d'érosion glaciaires (stries, cannelures) sont particulièrement bien préservées sur les supports peu sensibles à l'altération chimique ou à la dissolution que sont les grès du secteur Roche Noire-Roche Rousse par exemple (photo 1). La présence de ces stries et cannelures renseigne sur la direction de l'écoulement du glacier et suggère l'importance de son épaisseur. Ces microformes ne sont en revanche plus observables sur les gneiss.

\section{2 - Des formations glaciaires omniprésentes}

Les tills sont présents sur $15 \%$ de la surface étudiée (tableau 1). Dans le cirque du Lac Blanc, les tills sont essentiellement présents entre le glacier de Freydane et ses moraines de l'Holocène-PAG (figures 2 et 3 ), sous la forme de tills de placage mis en place à la base du glacier. Les incisions du torrent émissaire montrent une matrice fine abondante qui emballe des blocs rocheux faiblement émoussés. Ces premiers tills sont souvent surmontés de tills d'ablation mis en place lors des phases de recul du glacier, qui se distinguent des premiers par de moindres compaction et teneur en matrice fine. Les moraines latérales et frontales sont également constituées de till. En aval du complexe morainique de l'Holocène-PAG, des tills s'étendent jusqu'au Lac Blanc, séparés de celui-ci par un cône-delta torrentiel. Contrairement aux moraines plus récentes, la morphologie des moraines de l'Holocène-PAG est largement contrainte par la topographie puisque le glacier occupait alors tout le fond de la vallée. En particulier, la moraine latérale gauche se trouve perchée sur le versant. En aval de ces moraines, plusieurs cordons morainiques étagés, plus anciens, sont observables $200 \mathrm{~m}$ au nord du Lac Blanc. Des moraines que l'on peut raccorder à celles-ci sont présentes en rive droite du Ravin de la Lauzière de Roche Noire (moraines latérales) et au 


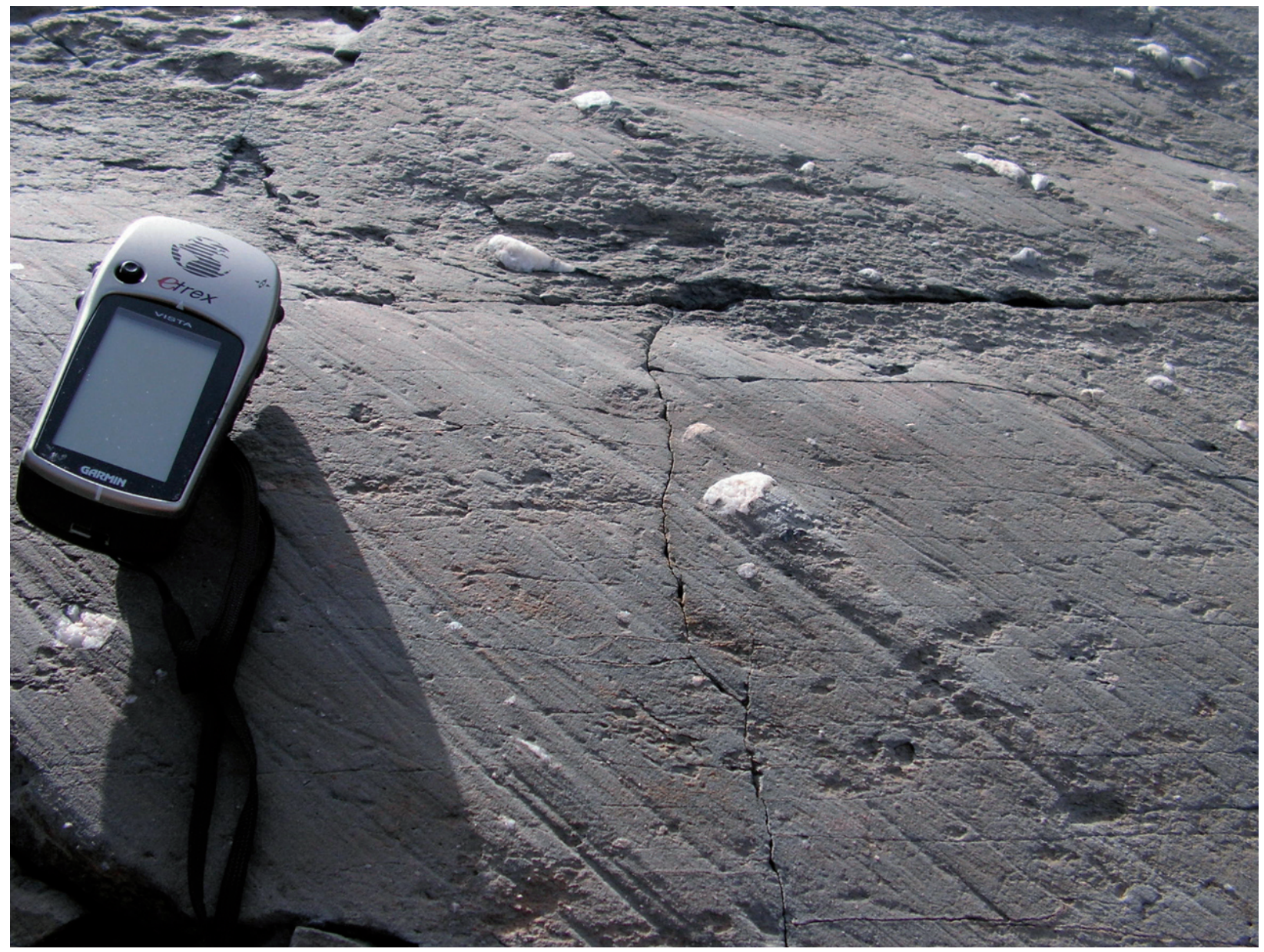

Photo 1 - Stries glaciaires et micro-queue de rat (au centre) sur les grès des roches moutonnées du Col de Roche Rousse. L'écoulement du glacier se faisait depuis l'angle en haut à gauche vers l'angle en bas à droite.

niveau du verrou du Lac Blanc (moraines frontales). Enfin, d'autres complexes morainiques perchés sur les versants sont également identifiables, notamment à l'ouest de Roche Rousse.

Dans le vallon de la Pierre, les complexes morainiques, pourtant très développés, sont plus difficiles à délimiter en raison de modelés souvent très émoussés, d'un important remaniement des tills par les processus périglaciaires, de dépôts d'écroulements recouvrant certaines moraines, ou encore de moraines ayant remanié des dépôts non-glaciaires, si bien que l'ensemble a pu être considéré comme un glacier rocheux (Chardon, 1993). Mais il s'agit bien de moraines, mises en place par un glacier très chargé en débris, comme l'indiquent des vallums morainiques très nets au sud de l'ensemble, qui se prolongent vers l'amont par des moraines émoussées mais individualisées, pour former enfin des moraines d'accrétion sur les marges latérales. Cette morphologie est différente de celle à rides et sillons transversaux qui caractérise un glacier rocheux. Ce sont ainsi 7 à 8 vallums morainiques qui peuvent être cartographiés entre $2070 \mathrm{~m}$ et $2290 \mathrm{~m}$ d'altitude, dont le plus élevé correspond à un bastion morainique édifié sous le Rocher de l'Homme (figures 2 et 4).

\section{3 - Des formations périglaciaires parasitant la lecture des héritages glaciaires}

Très largement présents dans le haut bassin du Vorz, les dépôts périglaciaires et gravitaires (glaciers rocheux, lobes de gélifluxion, talus d'éboulis, dépôts d'écroulement) occupent $36 \%$ de la surface étudiée (tableau 1). Si des moraines sont parfois fossilisées par des talus d'éboulis, d'autres peuvent être déstructurées par des processus périglaciaires. On observe des éboulis fluants sur le versant nord du Rocher de l'Homme, attestés par des rides mais aujourd'hui inactifs, dominés par un petit glacier rocheux (figures 2 et 4 ).

La compréhension de la mise en place des dépôts d'écroulements et de leur rapport aux formations morainiques est en revanche plus délicate. Ils peuvent les recouvrir, ou au contraire être incorporés dans les moraines. Dans le vallon de la Pierre, un important dépôt occupe le fond du vallon sur $24000 \mathrm{~m}^{2}$, recouvrant en partie deux moraines frontales (figures 2 et 4). La forme du dépôt suggère qu'il provient du secteur des Trois Officiers - Rocher de l'Homme, sans que l'on puisse préciser davantage son origine puisqu'aucune cicatrice d'arrachement ne ressort clairement sur les 


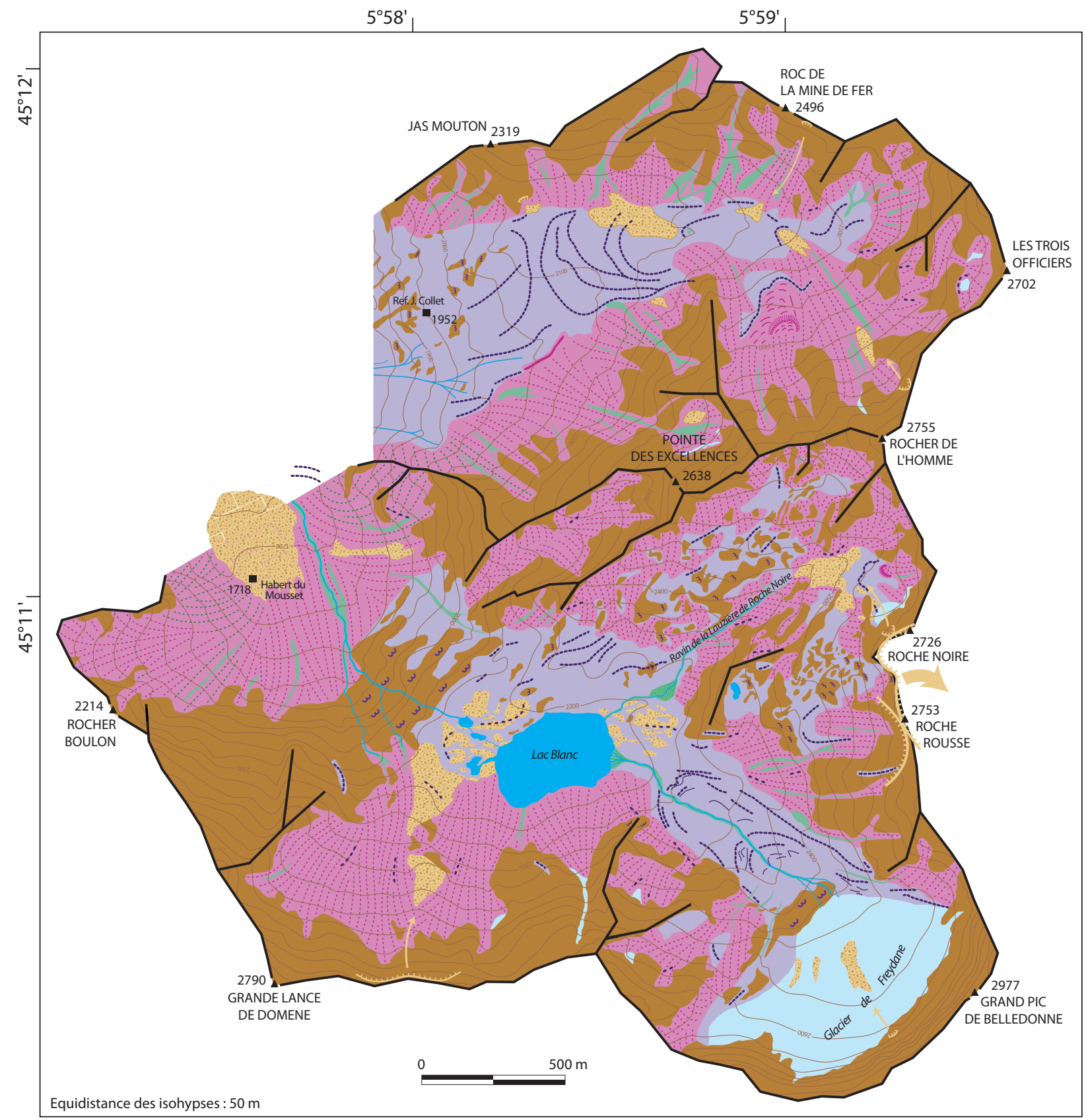

\begin{tabular}{|c|c|c|c|}
\hline$\Delta$ & Sommet principal & & $\begin{array}{l}\text { glacier } \\
\text { ou glacio-névé }\end{array}$ \\
\hline$\longrightarrow$ & ligne de crête & & tills \\
\hline & substratum rocheux & $\ldots \ldots$ & crête morainique \\
\hline & lac & $\ldots \ldots{ }^{\circ}$ & $\begin{array}{l}\text { crete moraıınique } \\
\text { présumée }\end{array}$ \\
\hline & cours d'eau & $3_{3} 3^{3}$ & roches moutonnées \\
\hline
\end{tabular}

Figure 2 - Carte géomorphologique du vallon de la Pierre et du cirque du Lac Blanc.

parois par ailleurs très accidentées. Les secteurs du cirque du Lac Blanc et de l'Habert du Mousset comprennent de nombreux dépôts d'écroulement. Le plus important est celui sur lequel est construit l'Habert, vers $1680 \mathrm{~m}$ d'altitude (figures 2 et 5). Sa superficie de $95000 \mathrm{~m}^{2}$ et son épaisseur moyenne probablement bien supérieure à $10 \mathrm{~m}$ suggèrent un volume supérieur à 1 million de $\mathrm{m}^{3}$. Il est difficile d'identifier l'origine de l'écroulement. On peut néanmoins formuler l'hypothèse de l'écroulement d'un sommet rocheux qui aurait 


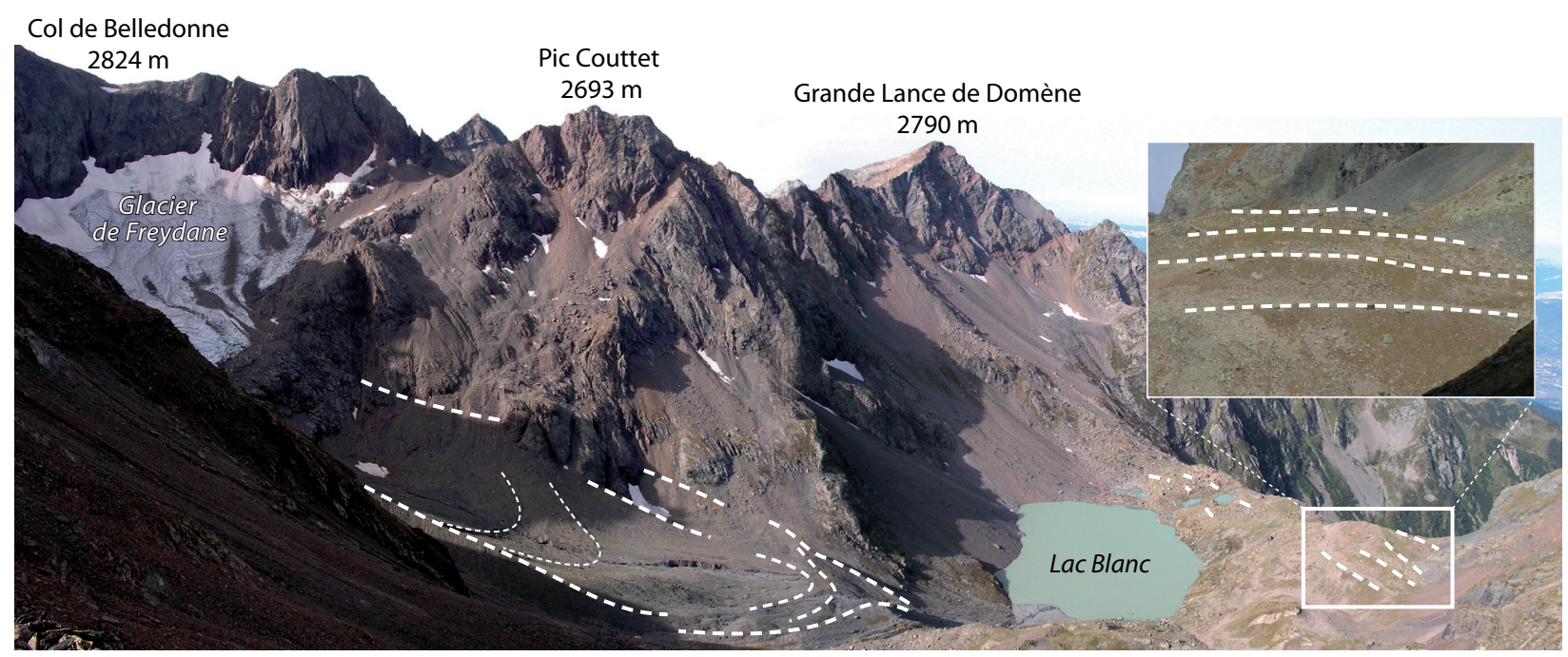

Figure 3 - Les moraines du PAG et post-PAG du glacier de Freydane, le lac Blanc et son verrou. En médaillon, quatre cordons morainiques bien identifiables au nord du lac Blanc.

\begin{tabular}{|c|c|c|c|c|c|c|c|c|c|c|}
\hline & & 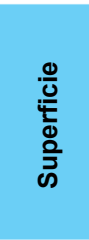 & 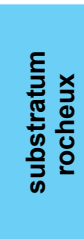 & 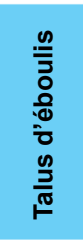 & $\stackrel{\varrho}{\equiv}$ & 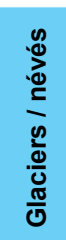 & 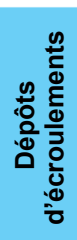 & 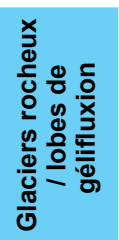 & జ⿱乛龰 & 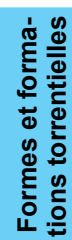 \\
\hline \multirow{2}{*}{$\begin{array}{l}\text { Vallon de } \\
\text { la Pierre }\end{array}$} & ha & 2950 & 1280 & 1010 & 542 & 10 & 40 & 41 & 2 & 25 \\
\hline & $\%$ & 100 & 44 & 34 & 18 & 0,3 & 1,5 & 1,5 & $<0,1$ & 1 \\
\hline \multirow{2}{*}{$\begin{array}{l}\text { Secteur du } \\
\text { Lac Blanc }\end{array}$} & ha & 5850 & 2464 & 1880 & 814 & 310 & 217 & 3 & 118 & 44 \\
\hline & $\%$ & 100 & 42 & 32 & 14 & 5,5 & 3,5 & $<0,1$ & 2 & 1 \\
\hline \multirow{2}{*}{ Total } & ha & 8800 & 3754 & 2890 & 1356 & 310 & 257 & 44 & 120 & 69 \\
\hline & $\%$ & 100 & 43 & 33 & 15 & 3,5 & 2,5 & 0,5 & 1,5 & 1 \\
\hline
\end{tabular}

Tableau 1 - Emprise spatiale des différentes composantes du haut bassin versant du Vorz.

été situé entre Roche Rousse et Roche Noire, précipitant un très grand volume (plusieurs dizaines de millions de $\mathrm{m}^{3}$ ?) sur le versant est. Le très grand dépôt $\left(>16000 \mathrm{~m}^{2}\right)$ présent dans le secteur de la Balmette, à $1,5 \mathrm{~km}$ à l'ouest du col de Roche Rousse, pourrait correspondre à une part importante du volume précipité sur ce versant. Le dépôt d'écroulement de l'Habert du Mousset correspondrait alors à la part (réduite) de l'écroulement qui aurait parcouru le versant ouest. Cette hypothèse s'appuie sur plusieurs éléments :

- la lithologie du secteur correspond à celle du dépôt (conglomérats, grès et schistes);

- le versant exposé à l'est est aujourd'hui très raide (présence de surplombs) et échancré (versant non raboté par les glaciers) ;

- des stries glaciaires et des micro-queues de rat sont présentes en contrebas de l'arête actuelle, ainsi que de nombreuses roches moutonnées sur le versant ouest du col de Roche Rousse, ce qui nécessite l'existence d'un épais paléo-glacier.

La surface du glacier régional étant située plus de $1000 \mathrm{~m}$ plus bas lors du Dernier Maximum Glaciaire, ce glacier ne pouvait être que local, mais son existence aurait été impossible avec la topographie actuelle. Si cette hypothèse concernant le dépôt du Habert du Mousset est avérée - ce que les dépôts à l'est du Lac Blanc et sur son verrou tendent à renforcer -, la distance horizontale parcourue par le volume rocheux aura été de $2750 \mathrm{~m}$, pour une dénivellation de $1100 \mathrm{~m}$.

Deux kilomètres à l'est de ce dépôt s'étend un autre dépôt d'écroulement, plus réduit $\left(2000 \mathrm{~m}^{2}\right)$, en aval des moraines PAG du glacier résiduel de Roche Noire (figures 2 et 6). La cicatrice d'arrachement sur la face NW de Roche Noire forme un grand plan de glissement $\left(2000 \mathrm{~m}^{2}\right.$; pendage $\left.35^{\circ}\right)$ surmonté par un escarpement légèrement surplombant (rupture par glissement banc sur banc). Ses dimensions suggèrent un volume écroulé de 15 à $20000 \mathrm{~m}^{3}$. Sur le glacier de Freydane enfin, en position presque centrale, un dépôt très récent de près de $5000 \mathrm{~m}^{2}$ s'étend au pied de la muraille des Trois Pics (figures 2 et 7). La cicatrice d'arrachement est située quelques dizaines de mètres au-dessus du glacier, à proximité immédiate de la brèche Reynier, et suggère un volume écroulé de l'ordre de $4500 \mathrm{~m}^{3}$. 


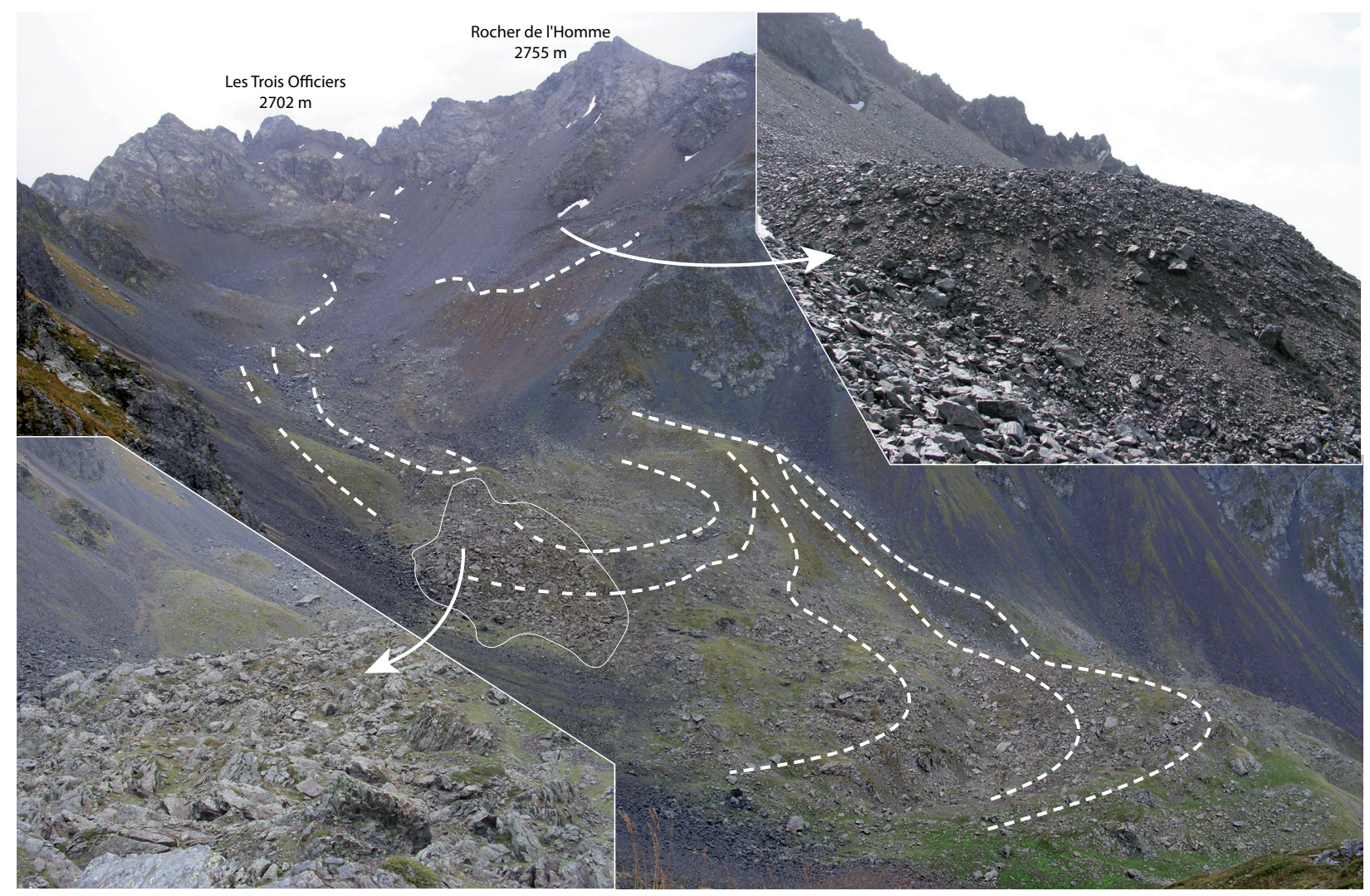

Figure 4 - Les moraines du vallon de la Pierre (tirretés) et les limites d'un dépôt d'écroulement (trait continu). La flèche en haut indique un glacier rocheux.

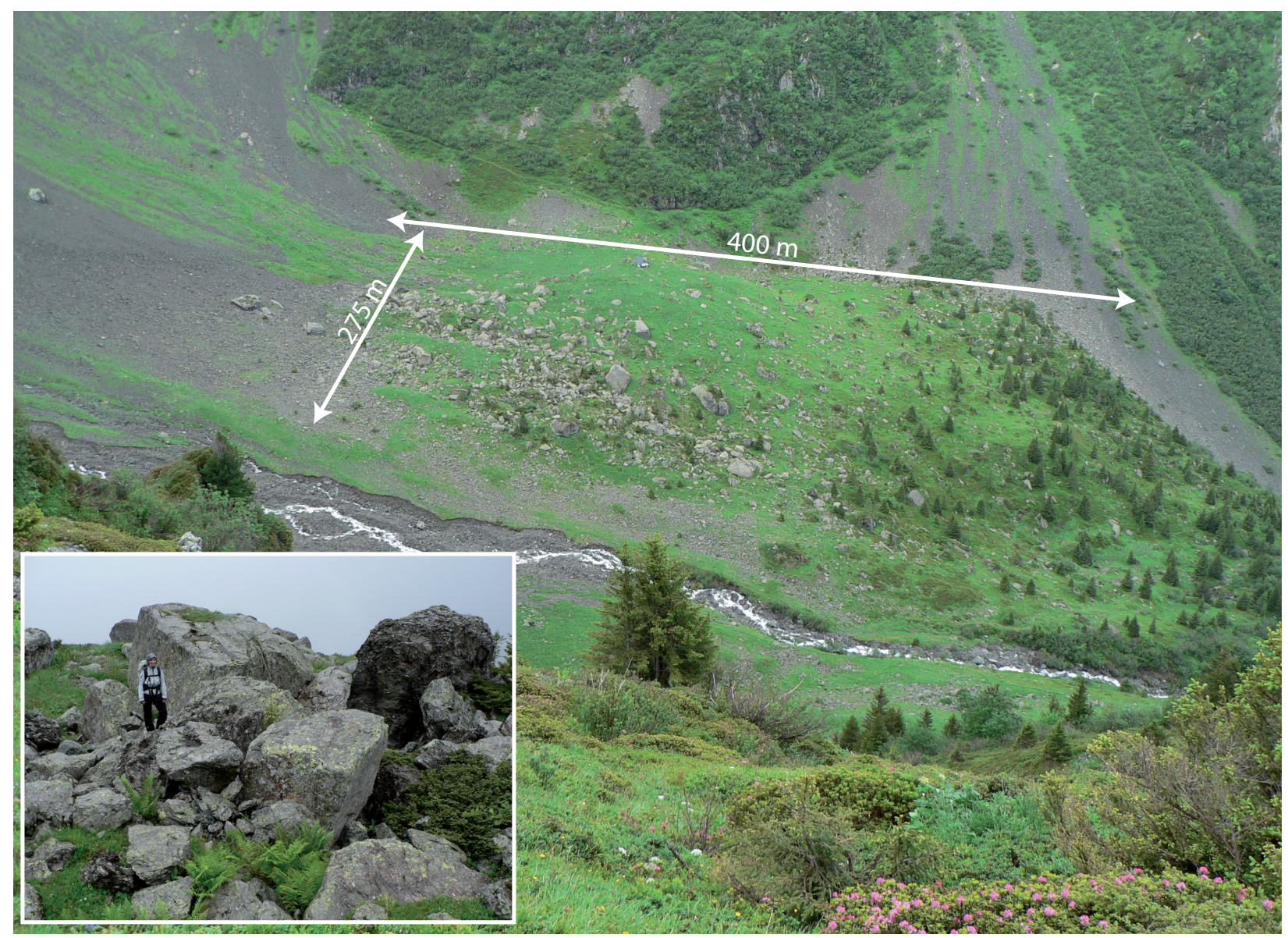

Figure 5 - Le dépôt d'écroulement de l'Habert du Mousset. En médaillon, la topographie très chaotique du dépôt. 


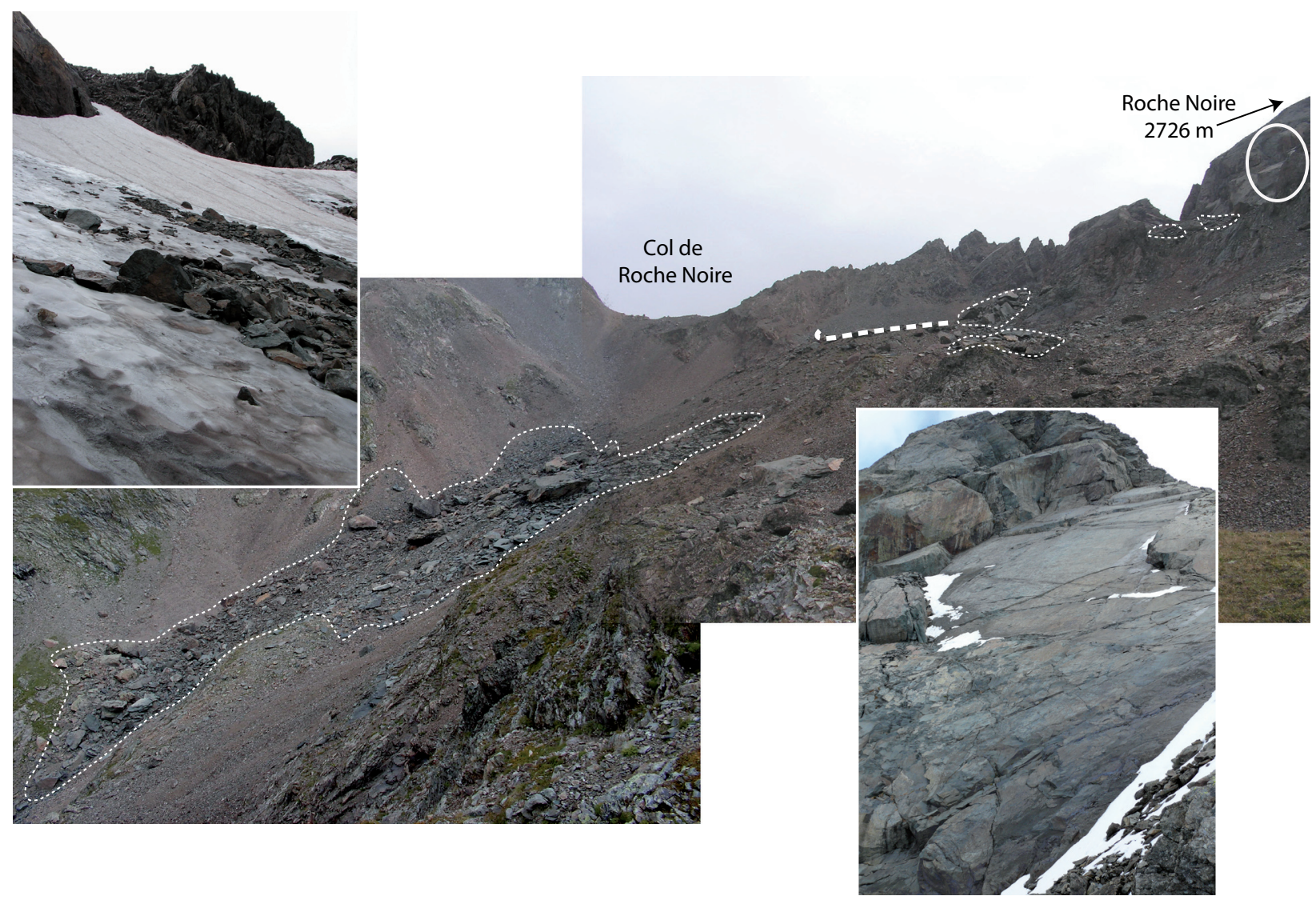

Figure 6 - Le dépôt d'écroulement de Roche Noire (pointillés). Tireté : moraine PAG du glacier de Roche Noire ; médaillon gauche : reliques actuelles du glacier de Roche Noire; cercle blanc et médaillon droit : cicatrice de l'écroulement.

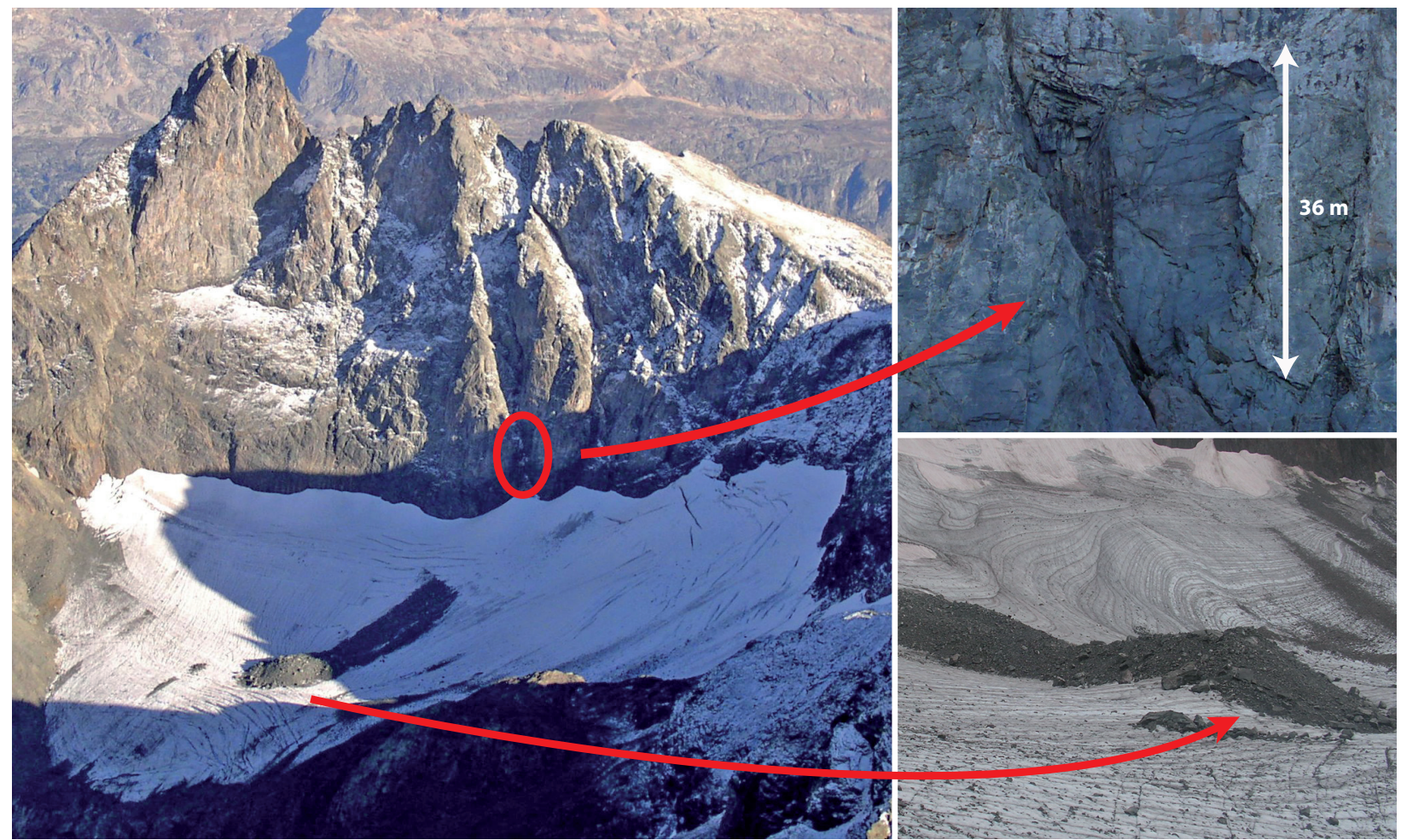

Figure 7 - L'écroulement de la face nord des Trois Pics. 


\section{III - RECONSTITUTION PALÉOGÉOGRAPHIQUE ET MORPHODYNAMIQUE DU HAUT VORZ}

Une fois les principales formes d'accumulation glaciaires et celles liées à des écroulements rocheux distinguées (figures 2), elles peuvent être calées dans une chronologie relative (Le Darz et al., 2004). A partir des moraines reportées sur la carte géomorphologique, il est d'autre part possible de reconstituer l'extension des glaciers pour les différentes positions correspondantes (Schoeneich, 1998 ; figure 9). Il devient alors possible de corréler les positions des appareils glaciaires de Freydane et du vallon de la Pierre et de raccorder cette chronologie à des chronologies absolues existantes à partir du calcul de l'altitude de la ligne d'équilibre (LEG) et de sa dépression (DEP) par rapport à la référence du maximum d'extension du Petit Age Glaciaire (1850), pour chacune des positions de ces glaciers. La ligne d'équilibre glaciaire correspond à la limite entre les zones d'accumulation et d'ablation, au bilan de masse glaciaire nul. Le calcul de son altitude (tableau 2) a été fait par la méthode de l'AAR (Accumulation Area Ratio) avec le rapport classique de 0,67 (Gross et al., 1978).

Si la chronologie des moraines s'établit en fonction de leur position, de leur fraîcheur, de leur altitude et du calcul des LEG et des DEP, celle des dépôts d'écroulements se base principalement sur leurs relations avec les moraines (recouvrement et remobilisation).

\section{1 - Le haut Vorz au Tardiglaciaire}

Le Tardiglaciaire (de $c .18000$ à 11600 ans cal. BP) correspond à la dernière phase du Pléistocène récent et précède l'Interglaciaire actuel (Holocène). Au cours de cette ultime période du Würm, le climat se réchauffe globalement même s'il connaît des phases froides. Le retrait généralisé des glaciers qui en résulte présente plusieurs stades, à l'origine de moraines souvent bien identifiables dans le paysage (figures 3 et 4). Six stades principaux - Bühl, Steinach, Gschnitz, Clavadel, Daun et Egesen - ont été mis en évidence par Maisch (1981). Deux sont identifiés comme des récurrences : le Gschnitz (Schoeneich, 2003 ; Coutterand et Nicoud, 2005 ; Kerschner et Ivy-Ochs, 2008) et l'Egesen, correspondant au maximum des oscillations du Dryas récent (Dorthe-Monachon, 1986).

$\mathrm{Au}$ moins sept voire huit positions tardiglaciaires, déterminées à partir des moraines situées en aval des moraines du PAG, peuvent être identifiées dans le secteur étudié (figure 8). En l'absence de marqueurs géomorphologiques nets, l'évolution des petits glaciers est plus délicate à reconstituer. A la position 13 - la plus ancienne position cartographiée -, les glaces issues du versant nord de la Pointe des Excellences et le glacier du vallon de la Pierre confluaient avec le glacier de Freydane. Le front de ce qui était alors le glacier du
Vorz descendait probablement jusqu'à l'actuel hameau de La Gorge, vers $730 \mathrm{~m}$ d'altitude, où une importante moraine frontale a été cartographiée par Chardon (1993). Entre la position 13 et la position 12, une ou plusieurs positions intermédiaires ont probablement été occultées, mais elles sont trop mal contraintes faute de moraines frontales cartographiées et malgré les moraines situées à l'ouest du refuge J. Collet. La position 12 est celle de l'individualisation des glaciers des deux bassins, ainsi que du petit glacier du versant nord de la Grande Lance de Domène (figure 8). Les deux cordons morainiques frontaux situés $400 \mathrm{~m}$ environ au NNE de l'Habert du Mousset, vers $1680 \mathrm{~m}$ d'altitude, peuvent être rattachés aux positions 12 et 11 . Les positions 11, $10,9,8$ et 7 , très proches (non cartographiées sur la figure 8), correspondent à des moraines intermédiaires du vallon de la Pierre (figure 9) ou situés au Nord du Lac Blanc. C'est entre les positions 8 et 7 que le grand dépôt d'écroulement du vallon de la Pierre (figure 4) s'est mis en place. Il repose en effet sur les moraines des positions 9 et 8 , tandis que sa partie proximale a été remaniée lors de la construction de la moraine frontale de la position 7 (figure 9). A la position 6, les glaciers de Roche Noire et de Freydane ne confluaient plus ; ce dernier se terminait alors probablement sur le verrou du Lac Blanc, à $2160 \mathrm{~m}$ d'altitude.

Selon le cadre chronologique relatif proposé ici, et au vu des DEP (tableau 2), il est possible d'effectuer des corrélations avec d'autres modèles de désenglacement de vallées alpines. Le calcul de l'altitude de la LEG du glacier du Vorz à la position $13(1860 \mathrm{~m})$ conduit à une DEP de $-725 \mathrm{~m}$. Cette valeur suggère le rattachement de cette position au stade de Gschnitz (antérieur à 15,4 $\pm 1,4$ ka BP ; Ivy-Ochs, 2008), reconnu dans le Gschnitztal (moraine de Trins) en Autriche. Il s'agit de la première réavancée significative post-würmienne des glaciers de montagne, alors que leurs fronts se situaient déjà à l'intérieur des massifs montagneux. Lors de ce stade, les glaciers ont avancé sur des distances considérables (Kerschner et al., 2002). Les moraines frontales qui auraient indiqué la position du front du glacier du Vorz aux stades de Clavadel et de Daun n'ont pas été reconnues dans cette étude : si ces stades ont existé, elles sont probablement situées dans la forêt de Saint Mury (Clavadel) et au Pleynet (Daun). La position 12, avec une DEP de $-320 \mathrm{~m}$, peut correspondre à la plus ancienne position (12,3 $\pm 1,5 \mathrm{ka} \mathrm{BP}$; Ivy-Ochs et al., 2008) du stade d'Egesen (Maisch, 1992 ; Ivy-Ochs et al., 2006). Les positions $11,10,9$ et 8 pourraient être rattachées à ce stade qui se caractérise par trois réavancées à moraines bien marquées. Les imposantes moraines frontales des positions 12,11 , et 9 du glacier du Vallon de la Pierre résultent probablement de ces réavancées ; les autres moraines, plus réduites, correspondraient à des stagnations. Avec une DEP de -195 m 


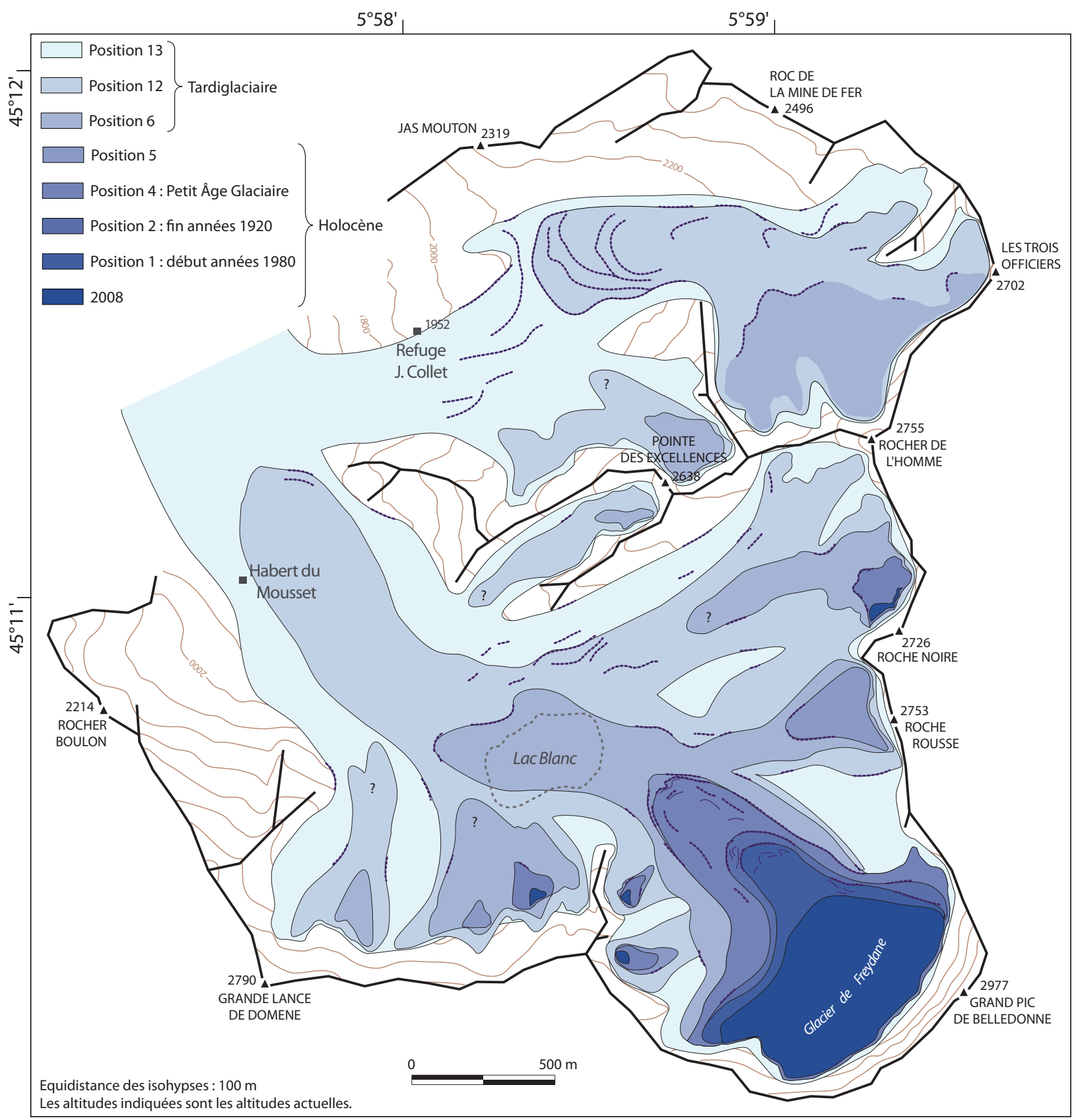

Figure 8 - Englacement du haut Vorz au Tardiglaciaire et à l'Holocène.

pour le glacier de Freydane, la position 6 peut indiquer une oscillation préboréale (stade de Kartell daté de 10,8 $\pm 1,0$ ka BP ? ; Ivy-Ochs et al., 2008). Cette position avait déjà été attribuée au Dryas récent (10 $000 \mathrm{BP})$ par Chardon (1993) sur la base des fluctuations relevées dans les Rousses (Chardon, 1991). Dans le Vallon de la Pierre, le calcul de la LEG du glacier à l'origine du bastion morainique massif situé vers $2280 \mathrm{~m}$ d'altitude suggère une formation contemporaine à celle des moraines de la position 6 du glacier de Freydane.

L'écroulement qui a engendré le dépôt de l'Habert du Mousset est probablement survenu à la fin du Tardiglaciaire ou au début de l'Holocène. En effet, le paléo-sommet de Roche Rousse n'a pas pu disparaître avant le Tardiglaciaire, période pendant laquelle a pu se développer le glacier local qui a érodé le substratum du secteur du col de Roche Rousse (roches moutonnées, stries et cannelures). D'autre part, comme le suggèrent la subverticalité et les nombreux surplombs du secteur amont du versant est de Roche Rousse, ainsi que l'absence d'émoussé, l'écroulement a dû avoir lieu à la fin de l'englacement local, soit au plus tôt à la fin du Tardiglaciaire. Enfin, le recouvrement des moraines latéro-frontales droites peut-être attribuables au Kartell (position 6) par des dépôts de cet écroulement indiquerait une occurrence au début de l'Holocène. 

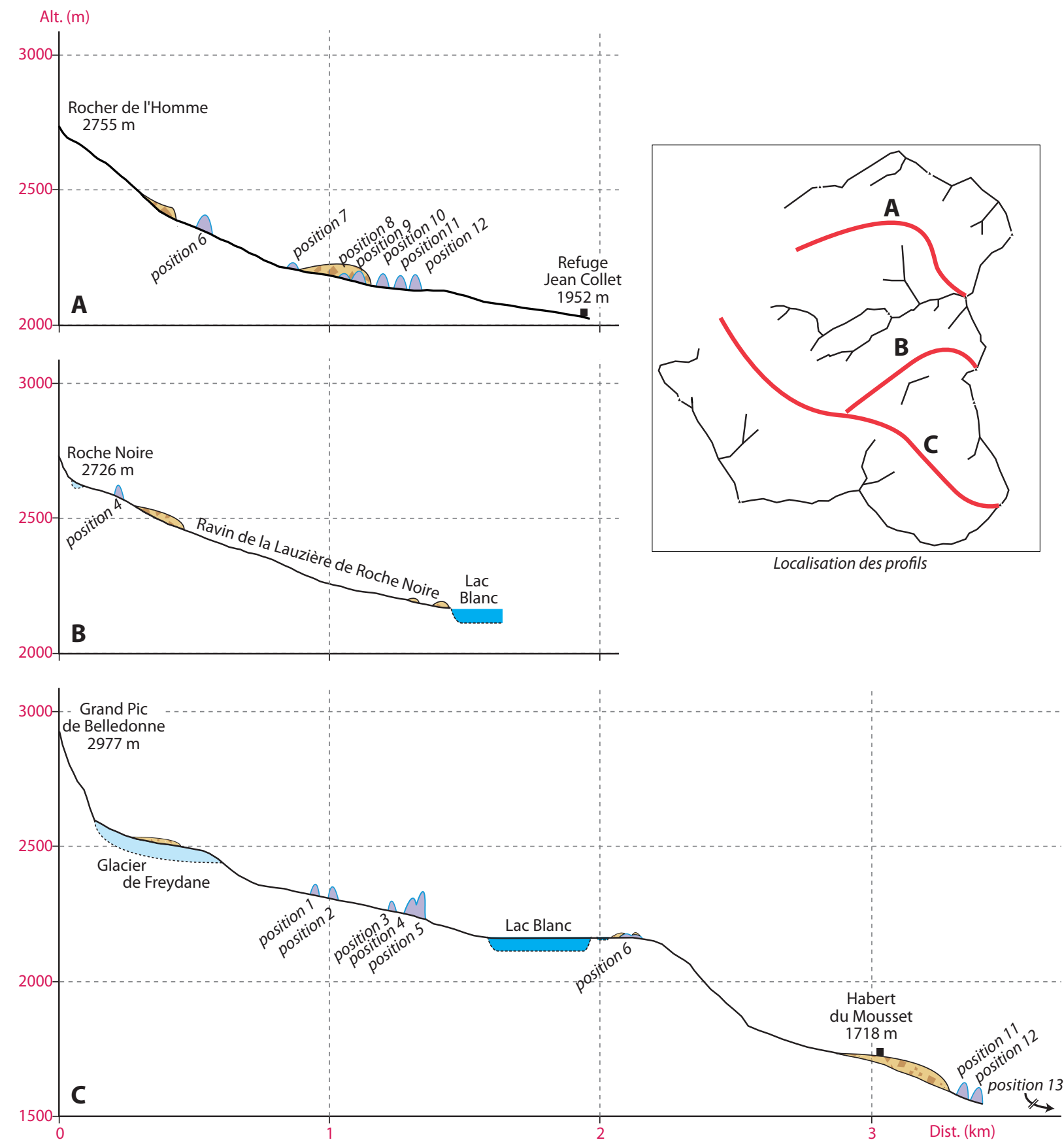

Figure 9 - Modèle de déglaciation du haut Vorz suivant trois profils topographiques (violet : moraines frontales; ocre : dépôts d'écroulements).

\section{2 - Evolution du haut Vorz durant l'Holocène}

Si la première moitié de l'Holocène, qui débute il y a 11600 ans, a vu un retrait généralisé des glaciers, la seconde (le Néoglaciaire) se caractérise au contraire par des réavancées glaciaires, généralement maximales lors du Petit Âge Glaciaire (Deline et Leroy, 2008). Bien que la date de son commencement soit encore débattue, le PAG court au moins du début du XVI $I^{\mathrm{e}}$ siècle jusqu'au milieu du XIX ${ }^{e}$ siècle (Grove, 1988), période caractérisée par une importante péjoration climatique (Le Roy Ladurie, 2004). Dans les Alpes, les avancées lors de la première moitié du XIX ${ }^{e}$ siècle correspondent, pour les glaciers petits et moyens, à l'extension maximale du PAG (Jobard, 2005). Elles ont donc fréquemment effacé les marqueurs géomorphologiques des positions précédentes du PAG voire des autres stades de l'Holocène. Des contre-exemples existent (e.g. Schneebeli et Roethlisberger, 1976), comme semble en être un le haut Vorz.

Dans le cirque du Lac Blanc, la crête morainique externe du bastion situé vers $2250 \mathrm{~m}$ d'altitude correspondrait à un maximum holocène antérieur au dernier maximum du PAG (position 5 ; figures 8 et 9 ) tandis 


\begin{tabular}{|c|c|c|c|c|c|c|c|}
\hline \multicolumn{2}{|c|}{ Position } & \multirow{2}{*}{$\begin{array}{c}\begin{array}{c}\text { Superficie } \\
\text { (km²) }^{2}\end{array} \\
8,75\end{array}$} & \multirow{2}{*}{$\begin{array}{l}\text { Superficie } \\
\text { / PAG (\%) } \\
+1200\end{array}$} & \multirow{2}{*}{$\begin{array}{c}\begin{array}{c}\text { Altitude } \\
\text { front }\end{array} \\
730\end{array}$} & \multirow{2}{*}{$\begin{array}{l}\text { Distance front } \\
\text { - moraine } \\
\text { PAG (m) } \\
-6330\end{array}$} & \multirow{2}{*}{$\begin{array}{l}\begin{array}{c}\text { Altitude LEG }(\mathrm{m}) \\
\text { [AAR] }\end{array} \\
1860 \pm 60\end{array}$} & \multirow{2}{*}{$\begin{array}{l}\text { DEP } \\
\text { (m) } \\
-725\end{array}$} \\
\hline P. 13 & GI. Vorz & & & & & & \\
\hline \multirow{2}{*}{ P. 12} & GI. Freydane & 2,78 & +380 & 1630 & -2250 & $2265 \pm 50$ & -320 \\
\hline & GI. Vallon Pierre & 0,77 & & 2080 & & $2250 \pm 50$ & \\
\hline \multirow{3}{*}{ P. 6} & Gl. Freydane & 1,06 & +145 & 2160 & -825 & $2390 \pm 30$ & -195 \\
\hline & GI. Roche Noire & $0,14 ?$ & $+360 ?$ & $2320 ?$ & $-650 ?$ & $2425 \pm 30 ?$ & $-165 ?$ \\
\hline & GI. Vallon Pierre & 0,38 & & 2280 & & $2410 \pm 30$ & \\
\hline \multirow{3}{*}{ P. 5} & GI. Freydane & \multicolumn{6}{|c|}{ Position quasi similaire à celle du PAG } \\
\hline & GI. Roche Noire & \multicolumn{6}{|c|}{ Idem } \\
\hline & GI. Vallon Pierre & \multicolumn{6}{|c|}{ Glacier disparu / Fonctionnement en éboulis fluant } \\
\hline \multirow{3}{*}{$\begin{array}{l}\text { P. } 4 \\
\text { PAG }\end{array}$} & GI. Freydane & 0,73 & & 2220 & & $2585 \pm 20$ & \\
\hline & GI. Roche Noire & 0,04 & & 2560 & & $2590 \pm 20$ & \\
\hline & GI. Vallon Pierre & \multicolumn{6}{|c|}{ Fonctionnement en glacier rocheux } \\
\hline \multirow{2}{*}{$\begin{array}{c}\text { P. } 2 \\
\text { Fin } \\
\text { années } \\
1920\end{array}$} & GI. Freydane & 0,46 & -36 & 2310 & +360 & $2630 \pm 10$ & +45 \\
\hline & GI. Roche Noire & \multicolumn{6}{|c|}{ Pas de témoignage géomorphologique } \\
\hline \multirow{2}{*}{$\begin{array}{l}\text { P. } 1 \\
\text { Début } \\
\text { années } \\
1980\end{array}$} & GI. Freydane & 0,43 & -42 & 2325 & +440 & $2635 \pm 10$ & +50 \\
\hline & Gl. Roche Noire & \multicolumn{6}{|c|}{ Pas de témoignage géomorphologique } \\
\hline \multirow{2}{*}{2008} & GI. Freydane & 0,34 & -53 & 2450 & +810 & $\begin{array}{c}\text { Quasi absence de } \\
\text { zone d'accumulation }\end{array}$ & $>100$ \\
\hline & GI. Roche Noire & 0,003 & -92 & 2615 & +130 & $\begin{array}{c}\text { Quasi absence de } \\
\text { zone d'accumulation }\end{array}$ & $>100$ \\
\hline
\end{tabular}

Tableau 2 - Evolution de l'englacement dans le haut bassin versant du Vorz.

que la crête interne, beaucoup plus fraîche et à colonisation végétale bien moindre, correspondrait à ce dernier, vers 1850 (position 4). Si quelques petits glaciers situés à l'ouest du glacier de Freydane ont construit des petits cordons morainiques durant l'Holocène et au PAG, le glacier de Roche Noire a lui édifié un important bastion morainique. Le calcul de la LEG de ce glacier pour le PAG place cette ligne à $2590 \mathrm{~m}$ d'altitude ( $2585 \mathrm{~m}$ pour le glacier de Freydane). La majeure partie de l'écroulement de Roche Noire (figure 6) a débordé ce bastion morainique, mais du matériel écroulé a été incorporé lors de la phase ultime de construction de la crête morainique - ce qui, avec la relative fraîcheur du dépôt, suggère que l'écroulement a eu lieu immédiatement avant la fin du PAG.

Dans le Vallon de la Pierre, les glaciers ont probablement disparu à la fin du Tardiglaciaire ou au début de l'Holocène, sans mettre un terme pour autant à la morphogenèse dans les secteurs antérieurement englacés. La dépression située à l'amont du bastion morainique supérieur est ainsi comblée par deux générations holocènes de formations périglaciaires : des éboulis fluants aujourd'hui inactifs (figure 2), surmontés par un petit glacier rocheux (figure 4) qui présente encore des signes d'activité (front très raide et instable, surface sans végétation y compris lichénique). Ce petit glacier rocheux pourrait s'être mis en place durant le PAG, comme dans d'autres régions (Osborn, 1975 ; Vere et Matthews, 1985).

Depuis la fin du PAG, les glaciers alpins subissent un recul généralisé (Vincent, 2002 ; Jobard, 2005). Si ce retrait n'est pas complètement achevé pour le glacier de Roche Noire, ses très faibles surface et épaisseur (tableau 1 ; figure 6) laissent présager une disparition totale dans les toutes prochaines années. La marge proglaciaire du glacier de Freydane illustre bien, quant à elle, sur plus de $800 \mathrm{~m}$, la décrue contemporaine des glaciers amorcée depuis la fin du PAG, non uniforme comme en témoignent les cordons morainiques entre le front actuel du glacier et les moraines PAG (figures 3,8 et 9). La plupart des glaciers des Alpes occidentales ont connu au moins trois réavancées depuis la fin du PAG, généralement vers 1890, dans les années 1910-20, et du milieu des années 1950 à celui des années 1980 (Deline et Le Roy, 2008). Une étude du nord du massif de Belledonne sur la période 1926- 
2006, fondée sur la télédétection, confirme que la fin des années 1920 et le début des années 1980 sont des périodes d'avancée des glaciers locaux au $\mathrm{XX}^{\mathrm{e}}$ siècle (Deroin et Condom, 2007), comme dans le massif du Mont-Blanc (Vincent et al., 2003). Les deux principaux vallums morainiques les plus récents (positions 2 et 1 ; figures 8 et 9) se mettent en place lors de ces deux périodes, comme le montrent cartes postales anciennes et cartes topographiques, tandis que la position 3 correspondrait à la récurrence de 1890, comme les photographies du début du $\mathrm{XX}^{\mathrm{e}}$ siècle le suggèrent. Le glacier de Freydane aurait ainsi perdu $53 \%$ de sa superficie entre la fin du PAG et 2007, avec un recul du front de $810 \mathrm{~m}$ (tableau 2).
Comme ailleurs dans les Alpes, le retrait du glacier de Freydane s'est certainement accéléré au cours de la dernière décennie, en particulier lors de l'été 2003. Ce glacier est aujourd'hui en situation de déséquilibre par rapport à son environnement. Il est intégralement situé en dessous de la LEG régionale et sa zone d'accumulation se cantonne aux cônes d'avalanches issues de la face nord des Trois Pics. Récemment, s'est également produit l'écroulement dont le dépôt repose aujourd'hui sur le glacier. La relation géométrique que présente la partie proximale du dépôt avec le névé sur les aérophotographies de la fin de l'été 2003 suggère une mise en place en 2001 : deux strates annuelles de névé ne sont pas recouvertes par le dépôt.

\section{Conclusion}

Durant le Tardiglaciaire et l'Holocène, le haut bassin versant du Vorz a connu une morphodynamique souvent intense, avec de nombreuses fluctuations glaciaires et de grands mouvements de versant. Sur la base d'une étude géomorphologique de ce haut bassin, treize positions glaciaires ont été identifiées. Contrairement à de nombreux secteurs des Alpes, des moraines holocènes antérieures au PAG n'ont pas été oblitérées lors de la dernière pulsation froide. Les quatre moraines les plus récentes peuvent être datées de 1850, de 1890, de la fin des années 1920 et du début des années 1980. Depuis la fin du PAG, les glaciers connaissent un fort retrait : plus de $300 \mathrm{~m}$ en 25 ans pour le glacier de Freydane, avec une probable accélération ces dernières années. Le glacier de Roche Noire n'est quant à lui plus que relique. A l'aval des complexes morainiques holocènes peuvent être distinguées au moins huit positions tardiglaciaires. Le calcul de la dépression des lignes d'équilibre glaciaire à partir de la cartographie des extensions des glaciers permet d'attribuer les positions correspondantes à des stades du modèle de référence pour les Alpes orientales (Maisch, 1982) ou septentrionales (Ivy-Ochs et al., 2008). Des techniques de datation isotopiques - ou lichénométriques pour la période récente (Jomelli, 1999) - permettraient de vérifier la chronologie proposée.

Enfin, cette étude a également permis de reconnâ̂tre quatre écroulements, d'âges et de dimensions très divers. L'écroulement principal, qui daterait de la fin du Tardiglaciaire ou du début de l'Holocène, correspond probablement à la disparition d'un paléo-sommet entre Roche Rousse et Roche Noire, et pourrait être lié au désenglacement du secteur.

\section{BibLIOGRAPHIE}

Allignol F. (dir.), 2008. Etude intégrée du bassin versant du Vorz (Belledonne, Isère) consécutive à la crue des 22 et 23 août 2005. Rapport scientifique, Laboratoire EDYTEM, Le Bourget du Lac, $202 \mathrm{p}$.

Campy M., Macaire J.-J., 2003. Géologie de la surface. Dunod, Paris, $440 \mathrm{p}$.

Chardon M., 1991. L'évolution tardiglaciaire et holocène des glaciers et de la végétation autour de l'Alpe d'Uez (Oisans, Alpes françaises). Revue de Géographie Alpine, 79, 39-52.

Chardon M., 1993. Glaciers et glaciers rocheux tardiglaciaires et holocènes de Belledonne (Alpes occidentales). In Géomorphologie et aménagement de la montagne. Hommage à P. Gabert, CNRS, Caen, 33-39.

Coutterand S., Nicoud G., 2005. Les stades de retrait du glacier de l'Arve entre le verrou de Cluses et l'ombilic de Chamonix au cours du Tardiglaciaire (vallée de l'Arve, Haute-Savoie). Quaternaire, 16, 85-94.
Deline P., Leroy M., 2008. Fluctuations des glaciers des Alpes occidentales depuis 5000 ans. Un état des connaissances. Collection EDYTEM, 6 - Cahiers de Paléoenvironnement, 9-24.

Deline P., KiRKBride, M.P., 2009. Rock avalanches on a glacier and morainic complex in Haut Val Ferret (Mont Blanc Massif, Italy). Geomorphology, 103, 80-92.

Deroin J.P., Condom T., 2007. Retrait séculaire des glaciers locaux de montagne : un exemple dans les Alpes occidentales françaises. Compte-Rendu Geoscience, 339, 449-459.

Dorthe-Monachon C., 1986. Contribution à l'étude de la morphologie glaciaire de la vallée de l'Arve (Haute-Savoie, France). Essai de reconstitution paléogéographique. Thèse, Université de Lausane, $242 \mathrm{p}$. 
Fernandez A., Guillot S., Menot R.-P., Ledru P., 2002. Late Paleozoic polyphased tectonics in the SW Belledonne massif (external crystalline massifs, French Alps). Geodinamica Acta, 15, 127-139.

Gross G., Kerschner H. et Patzelt G., 1978. Methodische Untersuchungen über die schneegrenze in alpinene Gletschergebieten. Zeitschrift für Gletscherkunde und Glazialgeologie, 12, 223-251.

Grove J.M., 1988. The Little Ice Age. Methuen, Londres, $498 \mathrm{p}$.

Guillot S., Ménot R.-P., 2009. Paleozoic evolution of the External Crystalline Massifs of the Western Alps. C. R. Geoscience, 341, 253-265.

Hanss C., Wegmüller S., 1976. Zur Altersstellung würmkaltzeitlicher Lokalgletschermoränen im Dévoluy und in der Belledonne (Französische Alpen). Zeitschrift für Gletscherkunde und Glazialgeologie, 12, 205-222.

Hippolyte J.-C., Tardy M., Nicoud G., 2006. Les failles récentes des Grands-Moulins (Savoie) : un Sackung (tassement gravitaire) majeur dans les Alpes francaises. Compte-Rendu. Geosciences, 338, 734-741.

Ivy-Ochs S, Kerschner H, Reuther A, Maisch M, Sailer R, Schaefer J, Kubik PW, Synal HA, Schlüchter C., 2006. The timing of glacier advances in the northern European Alps based on surface exposure dating with cosmogenic ${ }^{10} \mathrm{Be},{ }^{26} \mathrm{Al},{ }^{36} \mathrm{Cl}$, and ${ }^{21} \mathrm{Ne}$. In Siame L.L., Bourlès D.L. et Brown E.T. (éd.). In Situ-Produced Cosmogenic Nuclides and Quantification of Geological Processes, Special Paper 415. Geological Society of America: Boulder, 43-60.

Ivy-Ochs S., Kerschner H., Reuther A., Preusser F., Heine K., Maisch M., KubiK P.W., Schlüchter C., 2008. Chronology of the last glacial cycle in the European Alps. Journal of Quaternary Science, 23, 559-573.

JobARD S., 2005. Les glaciers du Haut Arc (Savoie) : caractérisation et impacts de la décrue post-Petit Âge Glaciaire. Thèse, Université de Savoie, Chambéry, 270 p.

Jomelli V., 1999. Les effets de la fonte sur la sédimentation de dépôts d'avalanches de neige chargée dans le massif des Ecrins (Alpes Françaises). Géomorphologie, 1, 39-58.

Kerschner H, Ivy-Ochs S, Schlüchter C., 2002. Die Moräne von Trins im Gschnitztal. Innsbrucker Geographische Studien, 33, 185-194.

Kerschner H., Ivy-Ochs S., 2008. Palaeoclimate from glaciers: examples from the Eastern Alps during the Alpine Lateglacial and early Holocene. Global and Planetary Change, 60, 58-71.
Le Darz M., Delannoy J.-J., Marnezy A. Nicoud G., 2004. La déglaciation du vallon de la Glière, massif de la Vanoise, Savoie. Travaux Scientifiques du Parc National de la Vanoise, 22, 7-21.

Le Roy Ladurie E., 2004. Histoire humaine et comparée du climat, 1, Canicules et glaciers (XIII ${ }^{\mathrm{e}}-\mathrm{XVIII}{ }^{\mathrm{e}}$ siècles). Fayard, Paris, 739 p.

MAISCH M., 1981. Glazialmorphologische und Gletschergeschichtliche Untersuchungen im Gebiet zwischen Landwasser-und Albulatal. Physische Geographie, 3, 215 p.

MAISCH M., 1982. Zur Gletscher- und Klimageschichte des alpinen Spätglazials, Geographica Helvetica, 2, 93-104.

MaISCH M. 1992. Die Gletscher Graubündens. Rekonstruktion und Auswertung der Gletscher und deren Veränderungen seit dem Hochstand von 1850 im Gebiet der östlichen Schweizer Alpen (Bündnerland und angrenzende Regionen). Physische Geographie, 33A/33B.

Osborn G., 1975. Advancing rock glacier in the Lake Louise Area, Banff National Park, Alberta. Canadian Journal of Earth Sciences, 12, 1060-1062.

Schneebeli W., Roethlisberger F., 1976. 8000 Jahre Walliser Gletschergeschichte. Les Alpes, CAS-SAC, 3-4.

Schoeneich P., 1998. Le retrait glaciaire dans les vallées des Ormonts, de l'Hongrin et de l'Etivaz (Préalpes vaudoises). Thèse - Travaux et Recherches de l'Institut de Géographie, Université de Lausanne, 14, 2 volumes, 483 p.

Schoeneich P., 2003. Que s'est-il passé pendant la première partie du Tardiglaciaire? Indices d'un changement écologique majeur dès 17-18 000 cal BP. Le Alpi : ambiente e mobilità : tavola rotonda, Trento, 25-27 ottobre 2001, 9-17.

Vere D. M., Matthews J. A., 1985. Glacier formation from a lateral moraine at Bukkeholsbreen, Jotunheimen, Norway: a sedimentological approach. Zeitschrift für Geomorphologie, 29, 397-415.

VINCENT C., 2002. Influence of climate change over the $20^{\text {th }}$ Century on four French glacier mass balances. Journal of Geophysical Research, 107(D19), 4375, doi:10.1029/2001JD000832.

Vincent C., Kappenberger G., Valla F., Bauder A., FunK M., Le Meur E., 2003. Ice ablation as evidence of climate change in the Alps over the $20^{\text {th }}$ Century. Journal of Geophysical Research, 109, 1-4. 



\section{NEIGE et GLACE de MONTAGNE \\ Reconstitution, dynamique, pratiques}

\section{Sommaire}

Editorial

Introduction

\section{1 - Reconstitution}

Coutterand S. et al. - Le lobe glaciaire lyonnais au maximum würmien : glacier du Rhône ou/et glaciers savoyards ?

Ravanel L. et al. - Désenglacement du haut bassin versant du Vorz (massif de Belledonne, Isère), au Tardiglaciaire et à l'Holocène.

Rey P.-J. - Sociétés et fluctuations du climat dans les Alpes nord-occidentales au Néolithique moyen.

Le Roy et al. - Étude des fluctuations glaciaires du Petit Âge de Glace dans le Massif des Écrins : apports de la lichénometrie.

Kirkbride M.P. - Datation des moraines holocènes d'Islande par tephrochronologie : un état de l'art.

Le Roy et al. - La dendroglaciologie, ou l'apport de l'étude des cernes d'arbres pour la reconstitution des fluctuations glaciaires holocènes.

Rabatel A. - Évolution glaciaire dans les andes subtropicales chiliennes entre 1955 et 2007 : conséquences pour la ressource en eau.

Le Roy et al. - Un inventaire des aérophotographies du massif du Mont Blanc.

\section{2 - Dynamique}

Ravanel L. - Évolution géomorphologique de la haute montagne alpine dans le contexte actuel de réchauffement climatique.

Gruber S. - Le permafrost de haute montagne.

Deline P. et al. - L'Aiguille du Midi (massif du Mont Blanc) : un site remarquable pour l'étude du permafrost des parois d'altitude.

Saulnier G.-M. et al. - Un éléphant volant est-il un oiseau ? Perspectives pour l'observation hydrométéorologique des milieux de montagne.

Jobard S. - L'instrumentation du glacier du Baounet : quels apports pour la traçabilité des mesures environnementales ?

Moreau L. - L'exploration du cryokarst glaciaire et son intérêt scientifique pour l'étude du drainage des eaux de fonte.

Mazué R. et al. - Suivi de l'évolution de la couverture detritique d'un glacier noir par photo-comparaison : le glacier d'Estelette.

\section{3 - Pratiques}

Paccard P. - Réchauffement climatique et ressource neige en domaines skiables.

Gauchon C. - Les hivers sans neige et l'économie des sports d'hiver : un phénomène récurrent, une problématique toujours renouvelée.

Laslaz L. - L'exclusion des glaciers des zones centrales des Parcs nationaux de la Vanoise et des Écrins et leur équipement pour le ski d'été.

Cayla N. - Les sentiers d'interprétation glaciaire : des outils de valorisation différenciée des glaciers et de leur territoire.

Lambert R. - Cartozonage : de la carte au zonage du risque avalanche.

Moulin A. et al. - L'incertitude liée aux avalanches dans les Alpes du Nord : identifications et implications pour la gestion.

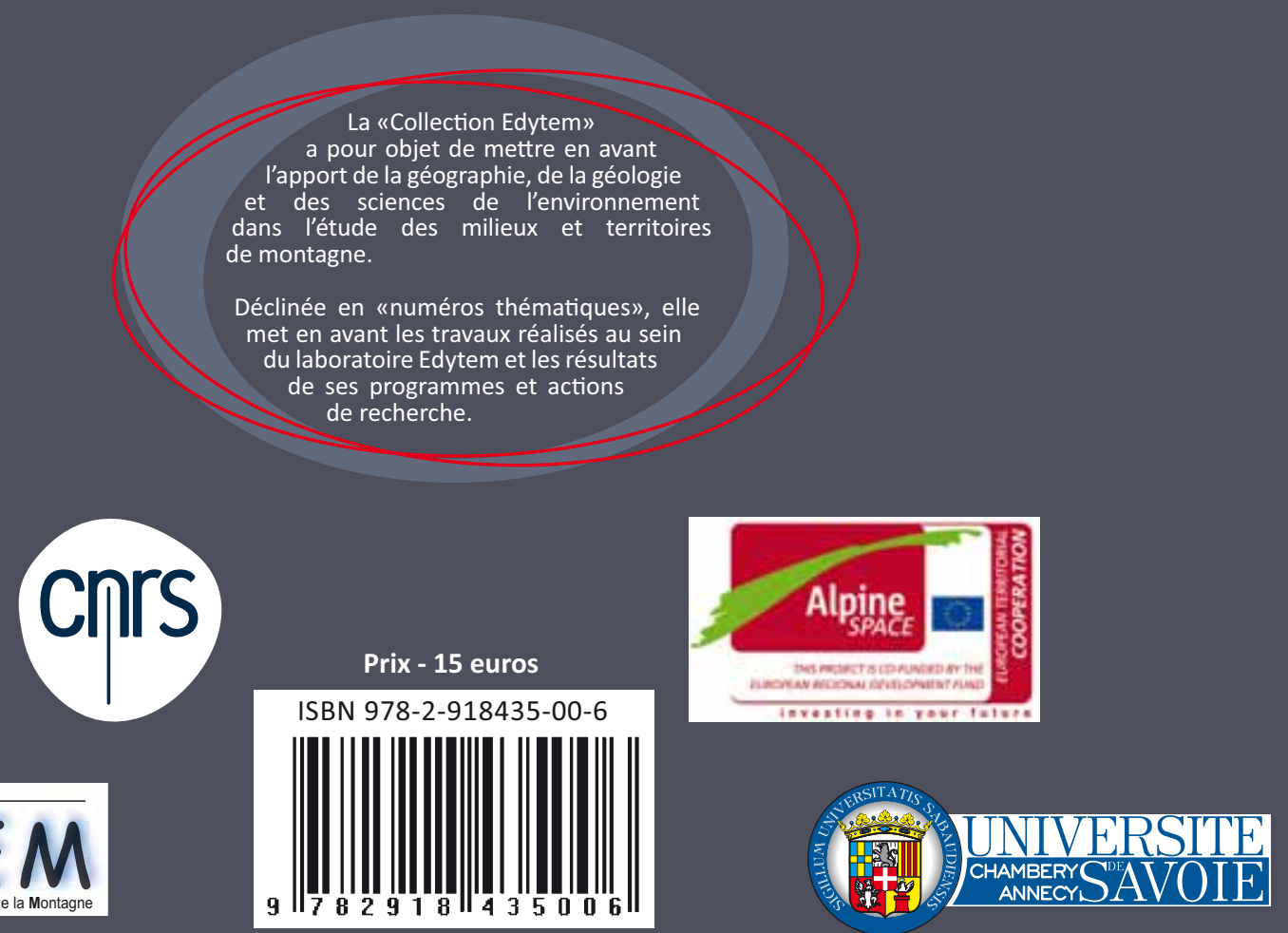

